\title{
Comparação do perfil comportamental de crianças com diferentes dermatoses crônicas
}

\section{Comparison of Behavioral Profile of Children with Different Chronic Skin Diseases Comparación del perfil comportamental de niños con diferentes dermatosis crónicas}

\author{
Natalia Guimarães Dias, Márcia Cristina Caserta Gon \\ Universidade Estadual de Londrina \\ Robson Zazula* \\ Universidade Federal da Integração Latino-Americana
}

Doi: http://dx.doi.org/10.12804/revistas.urosario.edu.co/apl/a.4506

\section{Resumo}

Objetivou-se comparar o perfil comportamental de crianças com doenças crônicas de pele (dermatite atópica, psoríase e vitiligo). Foi realizado um estudo transversal com uma amostragem por conveniência. Participaram 26 mães, com filhos entre seis e 12 anos e diagnóstico de doenças crônicas de pele. Os instrumentos utilizados foram o Child Behavior Checklist for ages 4/18 e o formulário sóciodemográfico. Realizou-se a comparação entre grupos de crianças com diferentes doenças crônicas de pele, quanto às competências sociais e problemas de comportamento, por meio do teste não paramétrico de Kruskal-Walis $(p<0,05)$. Os resultados indicaram diferenças estatisticamente significantes entre os três grupos quanto à competência global, social, escolar e para atividades. Crianças com dermatite atópica apresentaram menor competência para atividades e crianças com vitiligo para competência social. Os três grupos apresentaram baixa frequência de crianças com perfil clínico para competência escolar, e alta frequência de crianças classificadas como clínicas para problemas de comportamento. Discute-se o papel das dermatoses crônicas no desenvolvimento de problemas de comportamento e competências sociais. Palavras-chave: infância, adolescência, doenças crônicas de pele, problemas de comportamento.

\section{fbstract}

The main objective is to compare the behavioral profile of children with chronic skin disease (atopic dermatitis, psoriasis and vitiligo). A transversal study was conducted with a convenience sampling. Participants were 26 mothers, with children between the ages sis and 12 years and a chronic skin disease diagnosis. The instruments used were the Child Behavior Checklist for Ages 4/18 (CBCL 4/18) and a sociodemographic questionnaire. The results were compared between children groups with different chronic skin disease for social competences and problem behavior, using the non-parametric test of Kruskal-Walis $(\mathrm{p}<0.05)$.

* Correspondência: Correio eletrônico: robsonzazula@gmail.com

Cómo citar este artículo: Dias, N. G., Gon, M. C. C., \& Zazula, R. (2017). Comparação do perfil comportamental de crianças com diferentes dermatoses crônicas. Avances en Psicología Latinoamericana, 35(3), 559-570. doi: http://dx.doi.org/10.12804/ revistas.urosario.edu.co/apl/a.4506 
The results showed statistical differences between the three groups for global, social, activities and academic competences. Children with atopic dermatitis showed low competence to activities and children with vitiligo showed low social competence. All groups showed low frequencies of children classified as clinical for scholar competences and high frequencies of children classified as clinic for problem behavior. Discuses focused in the impact of chronic skin disease in the development of problem behavior and social competences.

Keywords: Childhood, adolescence, chronic skin disease, problem behavior.

\section{Resumen}

Se tuvo como objetivo comparar el perfil comportamental de niños con enfermedades crónicas de la piel (dermatitis atópica, psioriasis y vitiligo). Fue realizado un estudio transversal con un muestreo por conveniencia. Participaron 26 madres, con hijos entre 6 y 12 años y diagnósticos de enfermedades crónicas de la piel. Los instrumentos utilizados fueron el Child Behavior Checklist for ages 4/18 (CBCL 4/18) y el formulario sociodemográfico. Se realizó una comparación entre grupos de niños con diferentes enfermedades crónicas de piel en cuanto a las competencias sociales y problemas de comportamiento, por medio de la prueba no paramétrica de Kruskal-Walis $(\mathrm{p}<0,05)$. Los resultados indicaron diferencias estadísticamente significativas entre los tres grupos en cuanto a la competencia global, social, escolar y para actividades. Niños con dermatitis atópica presentaron menor competencia para actividades y niños con vitiligo para competencia social. Los tres grupos presentaron baja frecuencia de niños con perfil clínico para competencia escolar y alta frecuencia de niños clasificados como clínicas para problemas de comportamiento. Se discute el papel de las dermatosis crónicas en el desarrollo de problemas de comportamiento y competencias sociales.

Palabras clave: infancia, adolescencia, enfermedades crónicas de piel, problemas de comportamiento.
Dermatite atópica (DA), psoríase e vitiligo são doenças crônicas de pele, que têm como principais características o curso crônico e imprevisível. As doenças exigem rotinas de tratamento e cuidados constantes. A etiologia destas doenças ainda não é completamente conhecida e o uso de medicação tópica é necessário para o controle dos sintomas (Ahmed, Leon, Butler \& Reichenberg, 2013; Sampaio \& Rivitti, 1998).

Apesar de apresentarem características em comum, essas doenças se diferenciam quanto à forma das lesões, aos locais de manifestação dos sintomas no corpo e à rotina de tratamento médico. A DA afeta entre $10 \%$ e $20 \%$ das crianças em todo o mundo (Wall \& Sorensen, 2013; Yaghmaie, Koldelka \& Simpson, 2013) e se caracteriza por ser uma condição inflamatória crônica de pele com ocorrência de coceira, vermelhidão e lesões na pele em locais de intensa sudorese e/ou com articulações, tais como pescoço, joelhos e cotovelos (Berke, Singh \& Guralnick, 2012; Bieber, 2010; Silva \& Müller, 2007).

A psoríase afeta em média $1 \%$ da população infantil em todo o mundo (Mahé, Gnossike \& Sigal, 2014). Essa caracteriza-se pela ocorrência de lesões avermelhadas na pele, recobertas por escamas e, quando se manifesta na infância, acomete áreas como genitália, região glútea e periumbilical. Possui etiologia multifatorial e envolve fatores genéticos, imunológicos e ambientais (Mahé et al., 2014; Romiti, Maragno, Arnone \& Takahashi, 2009).

Por sua vez, o vitiligo caracteriza-se por ser uma doença crônica de pele assintomática, bem demarcada, cuja principal característica é a presença de manchas ou máculas acrômicas, com ausência total de pigmentação da pele (Palit \& Inamadar, 2012; Silva, Gontijo, Pereira \& Ribeiro, 2007; Silverberg, 2014). A prevalência do vitiligo na infância pode variar entre $0,1 \%$ e $4 \%$ da população, especialmente em meninas (Kanwar \& Kumaran, 2012).

Estudos têm investigado a relação entre doenças crônicas de pele, tais como a dermatite atópica, 
a psoríase, o vitiligo e a acne, com fatores como problemas psicológicos e transtornos psiquiátricos em crianças, adolescentes e adultos. Estresse, redução na qualidade de vida, ansiedade, tristeza, retraimento e dificuldades de interação social são os problemas mais comumente identificados nessas pesquisas (e.g., Berg, Svensson, Brandberg \& Nordlind, 2008; Kossakowska, Ciescinska, Jaszewska \& Placek, 2009; Ludwig, Oliveira, Müller \& Gonçalves, 2008; Ludwig, Oliveira, Müller \& Moraes, 2009; Magin, Sibbritt \& Bailey, 2009; Mota, Gon \& Gon, 2009; Perry \& Streusand, 2013; Yaghmaie et al., 2013). No entanto, existem poucos estudos e evidências empíricas conclusivas que demonstrem um perfil comportamental peculiar a indivíduos com doenças crônicas de pele, diferenciando-se conforme o tipo de doença, sobretudo durante a infância (Brufau et al., 2010; Kiebert et al., 2002; Perry \& Streusand, 2013; Rodriguez-Cerdeira, Pera-Grasa, Molares, Isa-Isa \& Arenas-Gusmán, 2011).

Apesar disso, observa-se que pais de crianças com DA relatam a presença de problemas de comportamento caracterizados como internalizantes (e.g., como ansiedade, depressão, queixas somáticas e isolamento social), e externalizantes (e.g., irritabilidade, desobediência e temperamento difícil; Fontes-Neto et al., 2005; Lewis-Jones \& Finlay, 1995; Pauli-Pott, Darui \& Beckmann, 1999; Perry \& Streusand, 2013; Rodriguez-Cerdeira et al., 2011). Segundo Yaghmaie et al. (2013), a probabilidade de desenvolvimento de transtorno de déficit de atenção e hiperatividade, ansiedade, depressão e problemas de comportamento é significativamente maior entre crianças com DA do que entre crianças sem a doença.

Em relação ao vitiligo, Choi, Kim, Whang, Lee, Hann e Shin (2010) constataram que diversas variáveis, tais como duração, severidade dos sintomas, manifestação da doença na face e história prévia de tratamento, influenciam significativamente no desenvolvimento da depressão, ansiedade e autoconceito negativo de crianças com a doença, mas não entre aquelas que não a apresentavam. Os autores discutiram, com base nesse resultado, que estas variáveis estão associadas à presença do vitiligo na amostra investigada. $\mathrm{O}$ estudo realizado por Krüger, Panske e Schallreuter (2014) constatou que crianças e adolescentes com vitiligo apresentaram maior dificuldade de interação interpessoal e estigmatização. A psoríase também foi associada à depressão e prejuízo da qualidade de vida em pacientes pediátricos (Bilgic, Bilgic, Akış, Eskioğlu \& Kılıç, 2010) e relacionou-se a um risco maior de desenvolver transtornos psiquiátricos, incluindo depressão e ansiedade, quando comparada com crianças em grupo controle sem a doença (Kimball et al., 2012).

Os resultados dos estudos realizados com crianças e adolescentes com dermatoses crônicas identificaram possíveis impactos negativos das doenças sobre o comportamento, saúde mental e qualidade de vida (Perry \& Streusand, 2013). Entretanto, esses foram obtidos a partir de investigações conduzidas predominantemente com um único tipo de doença (e.g., DA, psoríase ou vitiligo). Por essa razão, o presente estudo tem o objetivo de comparar o perfil comportamental de crianças com diagnóstico de diferentes dermatoses crônicas.

\section{Método}

\section{Participantes}

Amostra por conveniência composta por 26 mães, cujos filhos tinham idade entre seis e 12 anos, que participaram do projeto de extensão universitária para atendimento psicoeducacional a crianças com doenças crônicas de pele e seus pais e/ ou cuidadores, registrado na Pró-Reitoria de Extensão da Universidade Estadual de Londrina (PROEX n. ${ }^{\circ}$ 00952/2004). Os critérios para inclusão dos participantes no projeto foram: a) criança com alguma dermatose crônica, diagnosticada por médico dermatologista; b) busca por atendimento médico e/ou psicológico; e c) idade entre quatro e 12 anos. 


\section{Instrumentos}

Formulário de entrevista para triagem infantil. Coletar informações sobre a criança e familiares, tais como: a) nome; b) idade; c) sexo; d) escolaridade; e) dados residenciais; f) constituição familiar; e g) histórico de doença da criança.

Child Behavior Checklist for ages 4/18 (CBCL-4/18; Achenbach, 1991). Avaliar o comportamento das crianças quanto à a) competência social (e.g., competência total, atividades, escolares e sociabilidade); e b) problemas de comportamento (i.e., perfis internalizante e/ou externalizante). A versão utilizada foi a traduzida para o português (Brasil), por Bordin, Mari e Caiero (1995).

O instrumento é composto por 138 itens, dos quais 20 avaliam competência social e 118 problemas de comportamento. Nas questões relacionadas à competência social, investiga-se o envolvimento da criança ou adolescente em atividades diversas, participação em grupos, relacionamento com pessoas e autonomia. Ao responderem aos itens, os cuidadores devem comparar e classificar o comportamento de seus filhos com o de outras crianças ou adolescentes com a mesma idade.

Para a avaliação sobre problemas de comportamento, é apresentada uma lista contendo 118 comportamentos-problema, para os quais o cuidador é orientado a quantificar em uma escala de 0 a 2 pontos. Atribui-se para cada item ' 0 ', quando não é verdadeiro, ' 1 ', se é um pouco ou às vezes verdadeiro, e ' 2 ', se é muito ou frequentemente verdadeiro. Em ambas as avaliações, a criança é classificada como clínica, limítrofe ou não clínica.

\section{Procedimento}

Treinamento dos estudantes. Seis alunos do último período do curso de graduação em Psicologia foram treinados para realizar e preencher os formulários para entrevista de triagem infantil, aplicar e corrigir o CBCL. Além disso, os estudantes foram orientados para a divulgação, recrutamento dos participantes e realização das ações do projeto de extensão universitária com crianças com dermatoses crônicas e seus pais e/ou cuidadores.

Divulgação do projeto e recrutamento dos participantes. Realizada pelos estudantes, por meio de cartazes afixados em Unidades Básicas de Saúde e rede de transporte público do município de Londrina (PR), bem como no setor de Dermatologia do Ambulatório do Hospital de Clínicas da Universidade Estadual de Londrina (UEL). Além disso, foi realizada divulgação em programas de rádio e televisão, jornais impressos e portais de internet local. Os participantes que atenderam aos critérios de inclusão e procuraram o projeto de extensão universitária foram convidados para uma entrevista de triagem.

Realização da entrevista de triagem e aplicação do $C B C L$. Inicialmente, os estudantes explicaram aos participantes a proposta e os principais objetivos do projeto de extensão universitária. Os participantes que concordaram com os objetivos e aceitaram participar do projeto, leram e assinaram o Termo de Consentimento Livre e Esclarecido e, em seguida, foram entrevistados de acordo o formulário de entrevista de triagem.

Ao final da entrevista de triagem, os participantes foram orientados a preencher o CBCL. As entrevistas de triagem e aplicação do $\mathrm{CBCL}$ ocorreram individualmente na Clínica Psicológica da UEL e tiveram duração média de uma hora. A pesquisa, vinculada ao projeto de extensão universitária, foi aprovado pelo Comitê de Ética em Pesquisa Envolvendo Seres Humanos da UEL, sob parecer de aprovação $n^{\circ} 105 / 2010$.

Correção do CBCL. As informações coletadas foram corrigidas por meio do software ADM 7.0 (Assessment Data Manager). O software fornece a soma dos escores brutos e ponderados obtidos nas subescalas. A partir destes dados, podem ser obtidas três classificações do comportamento da criança (i.e., clínica, limítrofe e não clínica). 
Entretanto, neste estudo foram considerados em conjunto os casos limítrofes e clínicos. Isto ocorreu porque os escores limítrofe e clínico são incompatíveis com o esperado para a faixa etária em que as crianças se encontram. Dessa forma, os resultados serão descritos considerando as categorias clínica (CL) e não clínica (NC).

\section{Análise de dados}

Os dados obtidos por meio do formulário de entrevista e da correção do CBCL foram organizados e descritos em tabelas para análise. Em relação ao $\mathrm{CBCL}$, as comparações entre os grupos quanto as competências sociais e problemas de comportamento, foram realizadas por meio do teste não paramétrico de Kruskal-Walis, com valor de $p<0,05$.

\section{Resultados}

Em relação à caracterização sóciodemográfica $\mathrm{e}$ do tipo de dermatose apresentada pelas crianças, observou-se que 13 crianças (50\%) tinham diagnóstico médico de vitiligo, nove de DA $(34,6 \%)$ e quatro de psoríase $(15,4 \%)$. Considerando-se os três grupos (amostra total), 19 crianças eram do sexo feminino $(73,1 \%)$ e seis do sexo masculino $(26,9 \%)$. Entre as crianças do grupo DERM, sete eram do sexo feminino $(77,8 \%)$ e duas do sexo masculino $(22,2 \%)$. No grupo VIT, quatro eram do sexo masculino $(30,8 \%)$ e nove do sexo feminino (69,2\%). Entre as crianças do grupo PSO, uma era do sexo masculino (25\%) e três do sexo feminino (75\%).

Quanto à faixa etária, identificou-se no grupo DERM, predomínio de crianças entre 10 e 11 anos $(n=5 ; 55,5 \%)$; no grupo VIT entre 8 e 9 anos ( $n$ $=6 ; 46,2 \%)$ e no grupo PSO entre 8 e 9 anos $(n=$ $2 ; 50 \%)$. Com relação às características das mães dos três grupos, nove mães possuíam ensino médio (completo e incompleto; $34,6 \%$ ) e sete mães possuíam ensino fundamental (completo e incompleto; $30,7 \%$ ). No que tange à situação conjugal, 20 mães dos três grupos eram casadas $(76,9 \%$; tabela 1$)$.
Tabela 1

Características sóciodemográficas da amostra por tipo de dermatose crônica

\begin{tabular}{|c|c|c|c|c|}
\hline $\begin{array}{c}\text { Variáveis } \\
\text { sóciodemo- } \\
\text { gráficas }\end{array}$ & $\begin{array}{c}\text { DERM } \\
\mathrm{n}=9(\%)\end{array}$ & $\begin{array}{c}\text { VIT } \\
\mathrm{n}=13(\%)\end{array}$ & $\begin{array}{c}\mathrm{PSO} \\
\mathrm{n}=4(\%)\end{array}$ & $\begin{array}{c}\text { Amostra } \\
\mathrm{n}=26(\%)\end{array}$ \\
\hline \multicolumn{5}{|c|}{ Sexo da criança } \\
\hline Masculino & $2(22,2)$ & $4(30,8)$ & $1(25)$ & $7(26,9)$ \\
\hline Feminino & $7(77,8)$ & $9(69,2)$ & $3(75)$ & $17(73,1)$ \\
\hline \multicolumn{5}{|c|}{ Idade da criança } \\
\hline 6-7 anos & $0(0,0)$ & $2(15,4)$ & $1(25)$ & $3(11,6)$ \\
\hline 8-9 anos & $0(0,0)$ & $3(23,1)$ & $1(25)$ & $4(15,4)$ \\
\hline 10-11 anos & $1(11,1)$ & $1(7,9)$ & $0(0,0)$ & $2(7,7)$ \\
\hline 12 & $1(11,1)$ & $2(15,4)$ & $1(25)$ & $4(15,4)$ \\
\hline \multicolumn{5}{|c|}{ Escolaridade materna } \\
\hline $\begin{array}{l}\text { Ensino Fun- } \\
\text { damental }\end{array}$ & $1(11,1)$ & $5(38,5)$ & $2(50)$ & $8(30,7)$ \\
\hline $\begin{array}{l}\text { Ensino } \\
\text { Médio }\end{array}$ & $4(33,4)$ & $4(30,8)$ & $1(25)$ & $9(34,6)$ \\
\hline Superior & $4(33,4)$ & $4(30,8)$ & $1(25)$ & $9(34,6)$ \\
\hline \multicolumn{5}{|c|}{ Situação conjugal da mãe } \\
\hline Solteira & $1(11,1)$ & $0(0,0)$ & $0(0,0)$ & $1(3,9)$ \\
\hline Casada & $7(77,8)$ & $10(76,9)$ & $3(75)$ & $20(76,9)$ \\
\hline Divorciada & $1(11,1)$ & $3(23,1)$ & $1(25)$ & $5(19,2)$ \\
\hline
\end{tabular}

Nota . DERM $=$ Dermatite atópica; VIT $=$ Vitiligo; PSO $=$ Psoríase .

Fonte: Os autores.

Ao se avaliar a competência social da criança, observou-se maior quantidade de crianças consideradas clínicas no grupo DERM para competência total ( $n=5 ; 55,5 \%)$ e competência para atividades $(n=5 ; 55,5 \%)$, do que nos grupos viT $(n=$ $5 ; 38,5 \%$ para competência total e $n=2 ; 15,4 \%$ para atividades) e PSO $(n=1 ; 25,0 \%$ para competência total e $n=1 ; 25,0 \%$ para atividades). Tais diferenças foram consideradas estatisticamente significantes ao se comparar os três grupos quanto à competência total $[\mathrm{H}=3,57 ; \mathrm{gl}=2, p<0,05] \mathrm{e}$ competência para atividades $[\mathrm{H}=0,56 ; \mathrm{gl}=2$, $p<0,05]$. 
No grupo VIT, observou-se seis crianças consideradas clínicas $(46,2 \%)$ e sete não clínicas $(53,8 \%)$ para sociabilidade. Embora a frequência de crianças do grupo VIT consideradas clínicas tenha sido inferior à frequência das crianças consideradas não clínicas, esta foi mais elevada em relação aos grupos DERM $(n=2 ; 22,2 \%)$ e PSO $(n=1 ; 25,0 \%)$. Tais diferenças foram consideradas estatisticamente significantes ao se comparar os três grupos quanto à sociabilidade $[\mathrm{H}=1,75$; $\mathrm{gl}=2, p<0,05]$.

Quanto à competência escolar, foram identificadas frequências semelhantes de crianças clínicas para os grupos VIT $(n=4 ; 33,3 \%)$ e DERM $(n=3$; $33,3 \%$ ), e ligeiramente inferior no grupo PSO $(n=1 ; 25,0 \%)$. Tais diferenças foram consideradas estatisticamente significante ao se comparar os três grupos para esta competência $[\mathrm{H}=2,59$; $\mathrm{gl}=2, p<0,05]$. Os dados podem ser observados na tabela 2 , a seguir.

Tabela 2

Avaliação do CBCL quanto à competência social por tipo de dermatose crônica

\begin{tabular}{lccc}
\hline $\begin{array}{l}\text { Competên- } \\
\text { cias sociais }\end{array}$ & $\begin{array}{c}\text { DERM } \\
n=9(\%)\end{array}$ & $\begin{array}{c}\text { VIT } \\
\text { Total }\end{array}$ & $\begin{array}{c}\text { PSO } \\
n=4(\%)\end{array}$ \\
\hline CL & $\mathbf{5 ( 5 5 , 5 )}$ & $5(38,5)$ & $1(25,0)$ \\
NC & $4(44,5)$ & $\mathbf{8}(\mathbf{6 1}, \mathbf{5})$ & $\mathbf{3}(\mathbf{7 5}, \mathbf{0})$ \\
\hline Atividades & & & \\
\hline CL & $\mathbf{5 ( 5 5 , 5 )}$ & $2(15,4)$ & $1(25,0)$ \\
NC & $4(44,5)$ & $\mathbf{1 1}(\mathbf{8 4 , 6 )}$ & $\mathbf{3}(\mathbf{7 5 , 0 )}$ \\
\hline Sociabilidade & & & $1(25,0)$ \\
\hline CL & $2(22,2)$ & $6(46,2)$ & $\mathbf{3 ( 7 5 , 0 )}$ \\
NC & $\mathbf{7 ( 7 7 , 8 )}$ & $\mathbf{7 ( 5 3 , 8 )}$ & $1(25,0)$ \\
\hline Escolares & & & $\mathbf{3 ( 7 5 , 0 )}$ \\
\hline CL & $3(33,3)$ & $4(33,8)$ & $\mathbf{9 ( 6 9 , 2 )}$ \\
NC & $\mathbf{6 ( 6 6 , 7 )}$ & & \\
\hline
\end{tabular}

Nota . DERM $=$ Dermatite atópica $;$ VIT $=$ Vitiligo $;$ PSO = Psoríase; $\mathrm{CL}=$ Clínica; $\mathrm{NC}=$ Não Clínica.

Fonte: Os autores.
Foi encontrada maior frequência de crianças avaliadas com perfil clínico na escala global, independentemente do tipo de dermatose crônica (DERM $n=7 ; 77,8 \%$; VIT $n=7 ; 53,8 \%$; e PSO $n=3 ; 75,0 \%$ ). Tais diferenças foram consideradas estatisticamente significantes ao se comparar os três grupos $[\mathrm{H}=2,21 ; \mathrm{gl}=2, p<0,05]$. No entanto, ao se analisar cada um dos grupos, a maior frequência de crianças avaliadas como clínicas para o perfil internalizante ocorreu em DERM, com sete crianças (77,8\%), e em PSO, com três crianças $(75,0 \%)$. Tais diferenças foram consideradas estatisticamente significantes, ao se comparar os três grupos $[\mathrm{H}=2,57 ; \mathrm{gl}=2, p<0,05]$. O mesmo aconteceu ao se comparar os três grupos para o perfil externalizante. Seis crianças foram avaliadas como clínicas no grupo DERM $(66,6 \%)$ e três crianças no grupo PSO (75,0\%). Tais diferenças foram consideradas estatisticamente significantes ao se comparar os três grupos $[\mathrm{H}=3,07 ; \mathrm{gl}=2, p<0,05]$.

Tabela 3

Avaliação do CBCL quanto a problemas de comportamento por tipo de dermatose crônica

\begin{tabular}{lccc}
\hline $\begin{array}{c}\text { Problemas } \\
\text { de comporta- } \\
\text { mento }\end{array}$ & $\begin{array}{c}\text { DERM } \\
=9(\%)\end{array}$ & $\begin{array}{c}\text { VIT } \\
n=13(\%)\end{array}$ & $\begin{array}{c}\text { PSO } \\
n\end{array}$ \\
\hline Total & & \\
\hline CL & $\mathbf{7 ( 7 7 , 8 )}$ & $\mathbf{7 ( 5 3 , 8 )}$ & $\mathbf{3}(\mathbf{7 5 , 0 )}$ \\
\hline NC & $2(22,2)$ & $6(46,2)$ & $1(25,0)$ \\
\hline Internalizante & $\mathbf{7 ( 7 7 , 8 )}$ & $5(38,5)$ & $\mathbf{3 ( 7 5 , 0 )}$ \\
\hline CL & $2(22,2)$ & $\mathbf{8 ( 6 1 , 5 )}$ & $1(25,0)$ \\
\hline NC & & & \\
\hline Externalizante & $\mathbf{6 ( 6 6 , 7 )}$ & $5(38,5)$ & $\mathbf{3 ( 7 5 , 0 )}$ \\
\hline CL & $3(33,3)$ & $\mathbf{8 ( 6 1 , 5 )}$ & $1(25,0)$ \\
\hline NC &
\end{tabular}

Nota . DERM $=$ dermatite atópica $;$ VIT $=$ Vitiligo $;$ PSO $=$ Psoríase $; \mathrm{CL}$ $=$ Clínica; $\mathrm{NC}=$ Não Clínica.

Fonte: Os autores. 


\section{Discussão}

O resultado do presente estudo quanto à competência social de crianças com dermatose crônica, a partir da avaliação das mães pelo CBCL, sugere que aquelas do grupo DERM são menos competentes na realização de atividades do que as que possuem psoríase ou vitiligo, respectivamente. Esse dado pode ser explicado pelo fato de a DA exigir cuidados com a pele e que implicam em evitar situações que provoquem sudorese (e.g., práticas esportivas, brincadeiras ao ar livre), ressecamento da pele (e.g., natação) e contato direto com fatores ambientais precipitantes (e.g., poeira; Anthony, Gil \& Schanberg, 2003; Bieber, 2010; Silva \& Müller, 2007), os quais não são exigidos para as outras duas doenças. Portanto, é provável que, devido às restrições a tais situações com maior frequência, as crianças do grupo DERM envolvam-se menos em atividades esportivas e/ou de lazer do que as do grupo PSO e VIT e, por esta razão, se apresentem comparativamente menos competentes socialmente. Baixa competência para atividades também foi encontrada por Fontes-Neto et al. (2005) em crianças com DA, quando comparadas a um grupo controle de crianças sem a doença.

O segundo grupo com maior porcentagem de crianças que apresentaram baixa competência em atividades foi o PSO. Uma hipótese para explicar esse resultado poderia ser, não a restrição imposta para realização de atividades devido à possibilidade de aparecimento ou piora dos sintomas como ocorre na DA, mas sim ao desconforto físico causado pelos sintomas da psoríase como prurido, ardência, sensibilidade dolorosa das lesões e descamações. Consequentemente, as crianças com a doença participariam menos de atividades quando os sintomas estivessem presentes e o tratamento sendo conduzido. Portanto, dependendo da cronicidade da doença (i.e., recidiva e remissão dos sintomas), essas crianças teriam mais ou menos tempo de participação em atividades como nadar, brincar com bola, correr, entre outras. Portanto, é possível que os sintomas da dermatite atópica e da psoríase possam restringir a participação das crianças em atividades como as exemplificadas e, consequentemente, comprometer a qualidade de vida dos pacientes como destacaram Perry e Streusand (2013).

Por sua vez, as crianças do grupo viT foram as que mostraram ser mais competentes no desenvolvimento de atividades, o que pode ser justificado pelo tipo de sintoma que é a despigmentação da pele. Este exigiria apenas o uso de protetor solar e menos tempo de exposição ao sol, uma vez que a deficiência de produção da melanina deixa a pele mais sensível à radiação solar. Diferentemente da DA e da psoríase, o vitiligo é uma doença caracterizada pela despigmentação da pele (i.e., manchas brancas), sem que haja desconforto físico ou restrições para a realização de atividades (Rosa \& Natali, 2009). Assim, estes sintomas e cuidados com a pele não impedem que a criança participe de diversas atividades de lazer e esportivas, ao contrário do que pode acontecer com as do grupo DERM E PSO.

Apesar do grupo VIT ser o mais competente para atividades, este foi avaliado com mais crianças com perfil clínico para relacionamentos sociais, quando comparado aos grupos DERM e PSO. Enquanto diversos autores destacam baixo prejuízo para a apresentação de competências de relacionamento sociais entre crianças com DA (e.g., Fontes-Neto et al., 2005; Ludwig et al., 2009), maior dificuldade e problemas de socialização são esperados para crianças que apresentam vitiligo (Choi et al., 2010; Krüger et al., 2014), o que foi também observado no presente estudo. Tal dificuldade pode estar relacionada a uma baixa autoestima observada em muitos pacientes com vitiligo em virtude da condição inestética de sua pele e, consequente, estigmatização decorrente da doença. Sintomas generalizados pelo corpo, tipos de pele mais escuras, localização sobre a região do tórax e tratamentos anteriores malsucedidos, parecem ter um efeito negativo sobre aspectos psicossociais 
de indivíduos com vitiligo (Homan et al., 2009). Quando se tratam de adolescentes com a doença, Bilgic et al. (2010) observaram que o maior impacto psicossocial está diretamente relacionado com a maior severidade e localização da despigmentação da pele. Por outro lado, para Choi et al. (2010), a qualidade de vida de adolescentes com vitiligo está mais diretamente relacionada a apreensão quanto à doença, habilidade de ajustar-se psicossocialmente e presença de comorbidades psiquiátricas do que à severidade clínica da condição médica propriamente dita.

Quanto à competência escolar, observaram-se porcentagens baixas de crianças avaliadas como clínicas nos três grupos. Possivelmente, tal resultado demonstra pouca interferência das doenças no desempenho acadêmico das crianças (incluindo a investigação da nota/média das crianças nas disciplinas regulares, presença ou ausência de problemas de aprendizagem, repetência ou abandono e se frequentam ou não classes especiais). Embora sejam constatados prejuízos na realização de atividades acadêmicas, possivelmente problema está relacionado à desaprovação pelos pares e não a realização de atividades acadêmicas em si, como principal problema de crianças com doença de pele no contexto escolar.

Com base nos resultados sobre competência social, pode-se hipotetizar que os déficits comportamentais identificados nas crianças com dermatoses crônicas da amostra estudada possam ser considerados: a) como produto da condição biológica da pele (i.e., sintomas), uma vez que impõem restrições à participação em diversas atividades que possam ser prazerosas, auxiliar no desenvolvimento de habilidades físicas e favorecer a socialização; e/ou b) em função da exposição a situações aversivas (e.g., críticas, ofensas e chacotas), devido à condição médica a qual pode fazer com que muitas delas as evitem mais frequentemente. Esta fuga e/ ou esquiva das situações pode prejudicar a aprendizagem de comportamentos pró-sociais, que são importantes para o desenvolvimento emocional destas crianças.

Uma vez que se tratam de crianças e de adolescentes, os pais são os responsáveis pelos cuidados médicos que devem ser seguidos. É possível que, por haver uma preocupação maior com a condição médica de seu (sua) filho (a) com doença crônica, e por percebê-lo (a) com mais vulnerável, os pais limitem ainda mais sua participação em diversas atividades, temendo pela piora dos sintomas (Anthony et al., 2003), e também procurem evitar a exposição das lesões/manchas na pele, que podem ser alvo de preconceito. Qualquer que seja a razão, esta limitação poderá levar a criança a ficar mais retraída, explorar menos ambientes novos e diminuir a quantidade de interações sociais (Matos, 1983). Em consequência disso, a aprendizagem de habilidades físicas e sociais, importantes para o desenvolvimento infantil saudável, poderá ser prejudicada.

Nos três grupos foram encontradas maiores porcentagens de crianças avaliadas como clínicas do que como não clínicas na escala global e classificadas como clínicas para os perfis internalizante e externalizante. Este resultado sugere, assim como os obtidos em estudos sobre problemas de comportamento de pacientes pediátricos com doença crônica (e.g., Anthony et al. 2003; Bennett, 1994; Brufau et al., 2010; Castro \& Piccinini, 2002; Choi et al., 2010; Fontes-Neto et al., 2005; Krüger at al., 2014; Nelms, 1989; Yaghmaie et al., 2013), que crianças com dermatoses crônicas de pele podem ser mais susceptíveis a apresentar problemas de comportamento como ansiedade, depressão, isolamento social e agressividade (e.g., Perry \& Streusand, 2013).

Comparando-se os resultados entre os grupos para os perfis comportamentais, o grupo DERM apresentou maior porcentagem de crianças com perfil clínico para problemas do tipo internalizante e o grupo PSO apresentou porcentagem ligeiramente superior de crianças classificadas como clínicas para problemas de comportamento do 
tipo externalizante. O grupo VIT foi aquele que apresentou uma maior porcentagem de crianças avaliadas como não clínicas para esses dois perfis.

Resultados sobre comparação entre perfil comportamental de crianças com DA, psoríase e vitiligo ainda são inconclusivos, em especial pela escassez desse tipo de estudo com população pediátrica. De acordo com Rodriguez-Cerdeira et al. (2011), estudos que investigam a relação entre fatores psicológicos, distúrbios psiquiátricos e doenças dermatológicas ainda são escassos, por se tratar de uma área nova de pesquisa e estudos. Dentre os estudos existentes são comuns os que comparam crianças com doenças de pele com aquelas sem doenças. A pesquisa conduzida por Bilgic et al. (2010), por exemplo, encontrou que crianças com vitiligo são mais depressivas do que crianças não afetadas pela doença. Entretanto, Prćić et al. (2006) não encontraram diferenças quanto à apresentação de ansiedade e depressão entre adolescentes com vitiligo e quando comparadas com controles sem doença.

Independentemente dos resultados inconclusivos e do uso de instrumentos de medidas distintas (e.g., qualidade de vida, transtornos psiquiátricos, estresse, estigmatização) para estudar a mesma doença, há um consenso na literatura da área que destaca que diferentes doenças de pele podem causar graus variados de desconforto físico, psicológico e social (Ahmed et al., 2013; Perry \& Streusand, 2013; Rodriguez-Cerdeira et al., 2011). Assim, a relação entre a condição médica dermatológica e fatores psicossociais necessita ser investigada sistematicamente de modo a produzir conhecimento para subsidiar intervenções psicológicas e também de caráter social, direcionadas especificamente aos pacientes.

\section{Considerações finais}

Embora seja um estudo do tipo retrospectivo e com uma amostra por conveniência, feito por meio de um único documento padronizado e com amostra reduzida de participantes em cada grupo (sobretudo o grupo PSO), identificou-se características comportamentais relevantes apresentadas pelas crianças. Em síntese, o estudo demonstrou que: a) crianças com DA apresentam menor competência para atividades; b) crianças com vitiligo apresentam menor competência para sociabilidade; e c) nenhuma das doenças influenciam os resultados quanto à competência escolar.

Com relação aos problemas de comportamento, os resultados indicaram que há maior frequência de crianças avaliadas como clínicas do que não clínicas nos três grupos para a escala global, crianças com DA e psoríase apresentam problemas de comportamentos em maior frequência. Entretanto, não se pode concluir, mediante o delineamento conduzido nesta pesquisa, que os comportamentos apresentados pelas crianças e considerados problema ocorram em decorrência da doença em uma relação direta de causa e efeito. Certas condições dermatológicas, mais notadamente acne, DA e psoríase, podem ser exacerbadas pelo estresse e emoções. Por essa razão, causa e efeito podem se retroagir (Ahmed et al., 2013; Perry \& Streusand, 2013).

Devido, portanto, à complexidade das possíveis relações entre fatores psicológicos e condição médica dermatológica, como proposta de pesquisas futuras, sugere-se a realização de avaliações semelhantes à desta pesquisa, com maior controle de variáveis como maior número de participantes por grupo (ampliando assim o tamanho da amostra), identificação da localização e da quantidade das lesões, indicação do tempo de diagnóstico, entre outras.

Além disso, estudos com crianças e adolescentes com doença crônica de pele devem ser conduzidos. Embora os pais sejam importantes fontes de avaliação do comportamento de seus filhos e fundamentais no processo de intervenção médica e/ou psicoterapêutica, conhecer o que e como as crianças e os adolescentes percebem a doença e seu comportamento em relação a ela, é 
fundamental. Esse conhecimento torna a análise da interação, condição médica dermatológica e fatores psicológicos mais completa. No caso de pacientes com psoríase ou dermatite atópica, por exemplo, o efeito da doença no estado de ansiedade e de depressão tende a diminuir com o decorrer da idade, contudo fatores psicogênicos podem levar ao aparecimento ou à exacerbação dos sintomas dessas doenças, em especial durante a adolescência (Perry \& Streusand, 2013).

Considera-se também que adolescentes apresentem maturidade social e cognitiva para relatar seus sentimentos e comportamentos que ocorrem em diferentes situações de seu convívio. Há uma variação do $\mathrm{CBCL} / 6$-18 denominada Inventário de Autoavaliação para Adolescentes de 11 a 18 anos (YSR - Youth Self-Report; Achenbach, 2001). Esse faz parte do Sistema Integrado de Avaliação Empiricamente Baseado do Achenbach (Achenbach System Emprically Basead Assessment - ASEBA). As respostas obtidas por meio desta autoavaliação podem indicar qual a percepção desses indivíduos sobre suas competências e problemas de comportamento, e ainda ser comparada à de seus cuidadores. Como a metodologia utilizada em ambos os inventários são semelhantes há maior facilidade na comparação entre os dados obtidos a partir do relato dos pais ou responsáveis com o do adolescente (Achenbach \& Rescorla, 2001).

\section{Referências}

Achenbach, T. M. (1991). Manual for Child Behavior Checklist. Burlington: University of Vermont. Achenbach, T. M., \& Rescorla, L. A. (2001). Manual for the ASEBA school-age forms \& profiles. Burlington: University of Vermont, Research Center for Children, Youth \& Families.

Ahmed, A., Leon, A., Butler, D. C., \& Reichenberg, J. (2013). Quality-of-life effects of Common dermatological diseases. Seminars in Cutaneous Medicine And Surgery, 32(2), 101-109. doi:10.12788/j.sder.0009
Anthony, K. K., Gil, K. M., \& Schanberg, L. E. (2003). Brief report: parental perceptions of child vulnerability in children with chronic illness. Journal of Pediatric Psychology, 28(3), 185-190. doi:10.1093/jpepsy/jsg005

Bennett, D. S. (1994). Depression among children with chronic medical problems: a meta-analysis. Journal of Pediatric Psychology, 19(2), 149169. doi:10.1093/jpepsy/19.2.149

Berg, M., Svensson, M., Brandberg, M., \& Nordlind, K. (2008). Psoriasis and stress: a prospective study. Journal of the European Academy of Dermatology and Venereology, 22(6), 670-674. doi:10.1111/j.1468-3083.2008.02642.x

Berke, R., Singh, A., \& Guralnick, M. (2012). Atopic dermatitis: an overview. American Family Physician, 86(1), 35-42. Disponível em http:// www.aafp.org/afp/2012/0701/p35.html

Bieber, T. (2010). Atopic dermatitis. Annals of Dermatology, 22(2), 125-137. doi:10.5021/ ad.2010.22.2.125

Bilgic, A., Bilgic, Ö., Akış, H. K., Eskioğlu, F., \& Kılıç, E. Z. (2010). Psychiatric Symptoms and Health-Related Quality of Life in Children and Adolescents with Psoriasis. Pediatric dermatology, 27(6), 614-617. doi:10.1111/j.15251470.2010.01195.x

Bordin, I. A. S. J., Mari, J. J., \& Caiero, M. F. (1995). Validação da versão brasileira do "Child Behavior Checklist" (CBCL) (Inventário de Comportamentos da Infância e Adolescência): dados preliminares. Revista ABP-APAL, 17(2), 55-56.

Brufau, R. M., Berná, J. C., Redondo, C. B., Andreo, R. M. L., \& Gras, R. M. L. (2010). Estilos de personalidad en pacientes con psoriasis. Anales de Psicología, 26(2), 335-340. Disponível em http://www.um.es/analesps/v26/v26_2/1826_2.pdf

Castro, E. K., \& Piccinini, C. A. (2002). Implicações da doença orgânica crônica na infância para relações familiares: algumas questões teóricas. Psicologia: Reflexão e Crítica, 15(3), 625-635. doi:10.1590/S0102-79722002000300016 
Choi, S., Kim, D. Y., Whang, S. H., Lee, J. H., Hann, S. K., \& Shin, Y.J. (2010). Quality of life and psychological adaptation of Korean adolescents with vitiligo. Journal of the European Academy of Dermatology and Venereology, 24, 524-529. doi:10.1111/j.1468-3083.2009.03452.x

Fontes-Neto, P. T. L., Weber, M., Fortes, S., Cestari, T., Escobar, G., Mazotti, N. et al. (2005). Avaliação dos sintomas emocionais e comportamentais em crianças portadoras de dermatite atópica. Revista de Psiquiatria do Rio Grande do Sul, 27(3), 279-291. doi:10.1590/S010181082005000300007

Homan, M. W. L., Spuls, P. I., de Korte, J., Bos, J. D., Sprangers, M. A., \& van der Veen, J. W. (2009). The burden of vitiligo: patient characteristics associated with quality of life. Journal of the American Academy of Dermatology, 61(3), 411420. doi:10.1016/j.jaad.2009.03.022

Kanwar, A. J., \& Kumaran, M. S. (2012). Childhood Vitiligo: Treatment Paradigms. Indian Journal of Dermatology, 57(6), 466-474. doi:10.4103/0019-5154.103067

Kimball, A. B., Wu, E. Q., Guérin, A., Andrew, P. Y., Tsaneva, M., Gupta, S. R., et al. (2012). Risks of developing psychiatric disorders in pediatric patients with psoriasis. Journal of the American Academy of Dermatology, 67(4), 651-657. doi:10.1016/j.jaad.2011.11.948

Kiebert, G., Sorensen, S. V., Revicki, D., Fagan, S. C., Doyle, J. D., Cohen, D., \& Fivenson, D. (2002). Atopic dermatitis is associated with a decrement in health-related quality of life. International Journal of Dermatology, 41(3), 151158. doi:10.1046/j.1365-4362.2002.01436.x

Kossakowska, M. M., Ciescinska, C., Jaszewska, J., \& Placek, W. J. (2009). Control of negative emotions and its implication for illness perception among psoriasis and vitiligo patients. Journal of European Academy of Dermatology and Venerology, 23, 1050-1055. doi:10.1111/ j.1468-3083.2009.03432.x
Krüger, C., Panske, A., \& Schallreuter, K. U. (2014). Disease-related behavioral patterns and experiences affect quality of life in children and adolescents with vitiligo. International Journal of Dermatology, 53(1), 53-60. doi:10.1111/j.13654632.2012.05656.x

Lewis-Jones, M. S., \& Finlay, A. Y. (1995). The Children's Dermatology Life Quality Index (CDLQI): initial validation and practical use. British Journal of Dermatology, 132, 942-949. doi:10.1111/j.1365-2133.1995.tb16953.x

Ludwig, M. W. B., Oliveira, M. S., Müller, M. C., \& Gonçalves, A. M. B. F. (2008). Localização da lesão e níveis de estresse em pacientes dermatológicos. Estudos de Psicologia (Campinas), 25(3), 342-352.

Ludwig, M. W. B., Oliveira, M. S., Müller, M. C., \& Moraes, J. F. D. (2009). Qualidade de vida e localização da lesão em pacientes dermatológicos. Anais Brasileiros de Dermatologia, 84(2), 143 150. doi:10.1590/S0365-05962009000200007

Magin, P., Sibbritt, D., \& Bailey, K. (2009). The relationship between psychiatric illnesses and skin disease: a longitudinal analysis of young Australian women. Archives of Dermatology, 145(8), 896. doi:10.1001/archdermatol.2009.155

Mahé, E., Gnossike, P., \& Sigal, M.L. (2014). Le psoriasis de l'enfant. Archives de Pédiatrie, 21(7), 778-786. doi:10.1016/j.arcped.2014.04.018

Matos, M. A. (1983). A medida do ambiente de desenvolvimento infantil. Psicologia, 9(1), 5-18.

Mota, C. M. S., Gon, M. C. C. \& Gon, A. S. (2009). Análise comportamental de problemas de interação social de indivíduos com psoríase. Interação em Psicologia, 13, 155-164.

Nelms, B. C. (1989). Emotional Behaviors in Chronically Ill Children. Journal of Abnormal Child Psychology, 17(6), 657-668. doi:10.1007/ BF00917729

Palit, A. \& Inamadar, A. C. (2012). Childhood vitiligo. Indian Journal of Dermatology, Venereology and Leprology, 78(1), 30-41. doi:10.4103/03786323.90944 
Pauli-Pott, U., Darui, A., \& Beckmann, D. (1999). Infantis with atopic dermatitis: maternal hopelessness, child-rearing attitudes and perceived infant temperament. Psychotherapy Psychosomatic, 68, 39-45. doi:10.1159/000012309

Perry, M., \& Streusand, W.C. (2013). The Role of Psychiatry and Psychology Collaboration in Pediatric Dermatology. Dermatologic Clinics, 31(2), 347-55. doi:10.1016/j.det.2012.12.012 Prćić, S., Durović, D., Duran, V., Vuković, D. \& Gajinov, Z. (2006). Some psychological characteristics of children and adolescents with vitiligo: our results. Medicinski Pregled, 59(5-6), 265-269. doi:10.2298/MPNS0606265P

Rodríguez-Cerdeira, C., Pera-Grasa, J. P., Molares, A., Isa-Isa, R., \& Arenas-Guzmán, R. (2001). Psychodermatology: Past, Present and Future. The Open Dermatology Journal, 5, 21-27. doi:10.2174/1874372201105010021

Romiti, R., Maragno, L., Arnone, M., \& Takahasi, M. D. F. (2009). Psoríase na infância e adolescência. Anais Brasileiros de Dermatologia, 84(1), 9-22. doi:10.1590/S0365-05962009000100002

Rosa, E. C., \& Natali, M. R. M. (2009). Vitiligo: um problema que não pode passar em branco. Revista Saúde e Pesquisa, 2(1), 119-126.
Disponível em http://periodicos.unicesumar. edu.br/index.php/saudpesq/article/view/910/732 Sampaio, S. A. P. \& Rivitti, E. (1998). Dermatologia. São Paulo: Artes Médicas.

Silva, C. M. R., Gontijo, B., Pereira, L. B., \& Ribeiro, G. B. (2007). Vitiligo na infância: características clínicas e epidemiológicas. Anais Brasileiros de Dermatologia, 82(1), 47-51. doi:10.1590/ S0365-05962007000100006

Silva, J. D. T., \& Müller, M. C. (2007). Uma integração teórica entre psicossomática, stress e doenças crônicas de pele. Estudos de Psicologia (Campinas), 24(2), 247-256. doi:10.1590/ S0103-166X2007000200011

Silverberg, N. B. (2014). Pediatric vitiligo. Pediatrics Clinics of North America, 61(2), 347-366. doi:10.1016/j.pcl.2013.11.008

Wall, L. A., \& Sorensen, R. U. (2013). Atopic dermatites: looking beyond the skin. The Journal of Pediatrics, 163(6), 1547-1548. doi:10.1016/j. jpeds.2013.08.065

Yaghmaie, P., Koudelka, C. W., \& Simpson, E. L. (2013). Mental health comorbidity in patients with atopic dermatitis. Journal of Allergy and Clinical Immunology, 131(2), 428-433. doi:10.1016/j.jaci.2012.10.041 